\section{Cancer-associated thrombosis and palliative care: an interview with Simon Noble}

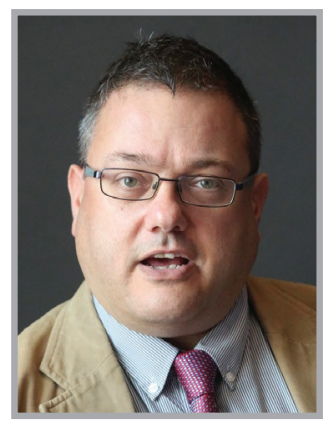

Simon Noble speaks to Sebastian Dennis-Beron, Commissioning Editor: Simon Noble is Clinical Professor in Palliative Medicine at Cardiff University and honorary consultant at the Royal Gwent Hospital in Newport. His main research interests are in the management of venous thromboembolism in advanced cancer, quality of life effects of venous thromboembolism and their therapies, clinical decision-making in venous thromboembolism management and the patient journey. He is involved at a national level in the delivery of evidence-based thromboprophylaxis for hospitalized patients through the All Party Thrombosis Group and sits on the NICE Guideline development group for thromboprophylaxis. He is a Medical Director of Thrombosis UK and is currently chairing the All Wales HAT Prevention Steering Group. Internationally he sits on the ISTH Cancer Scientific Sub Committee and American Society for Hematology Cancer Associated Thrombosis Guidelines. He has published over 100 original papers.

Accepted for publication: 27 September 2016; Published online: 19 October 2016

Q Can you tell us about your career? How did you become involved in cancer palliative care?

I am currently a Clinical Professor in Palliative Medicine and I have been qualified for 23 years. I became very interested in palliative care long before I qualified as a medical student on a general practice attachment. On one of the days it was arranged for me to go to a hospice. At this point I was a bit jaded in my medical career - I had lost my way a bit - so when I went to this hospice I found it pretty life changing. I thought to myself, here is where you can be a good general physician, you have got time for the patients, the family and I really liked the holistic approach.

So I had always had an interest in doing palliative medicine and ended up doing my elective in a hospice in Wolverhampton. It was very clear to me that you had to get a very extensive general medical experience first. So I did a lot of general medicine and it was during that time that I particularly remember one patient named Joyce. She was admitted in atrial fibrillation, and I remember from my studies that one of the causes of atrial fibrillation is a blood clot - a pulmonary embolism. I had also noticed that she was jaundiced and I put two and two together and thought, "this lady maybe had pulmonary embolism which has put her in atrial fibrillation, and she is jaundiced because she has a pancreatic cancer" and that was exactly what had happened.

This was the first time I appreciated that there was an association between malignancy and blood clots. From that point on, however, it was something I had a clinical interest with. I ended up working in palliative medicine in Cornwall, UK for some time, then

*Palliative Medicine, Marie Curie Palliative Care Research Centre, Cardiff University School of Medicine, 1st Floor, Neuadd Meirionyd Heath Park Way, Cardiff, CF14 4YS, UK; Simon.noble@wales.nhs.uk

\section{KEYWORDS}

- anticoagulation - blood clots cancer and you $\bullet$ cancer-associated thrombosis $\bullet$ CAT $\bullet$ heparins - palliative care $\bullet$ patient experience - PELICAN • thromboprophylaxis

- thrombosis • venous thromboembolism 
I realized I wanted to do a formal training program so I came up to Cardiff, UK, and it was there that I had this serendipitous meeting, with one of the consultants in the car park who had heard that I had an interest in cancer-associated thrombosis (CAT). He asked me what I thought would happen if we gave low-molecular weight heparin to lung cancer patients, and then I explained to him all the theories there were about all the potential anticancer effects of heparins and as a result, he suggested I write a protocol for this. A year later, we had landed a US\$1 million grant from Pfizer, and a GB£630,000 grant from Cancer Research UK to undertake the FRAGMATIC study which turned out to be, at the time, the largest lung cancer clinical trial in the world. It took us until last year to actually report it as it was a very large study, and that was the beginning of my academic career.

Now, over that time I spent a lot more time getting interested in CAT and it was very clear that we can do vast amounts about the pharmacology of the drugs we were using and the pathophysiology of the malignant thrombotic state, but there were a lot of assumptions being made about the patient and how the medicines were or were not acceptable to certain cancer patients. I did a lot of research looking at patient acceptability of heparins and attitudes to CAT, and that began to get me noticed within the CAT world.

\section{Q How has cancer palliative care developed over the past decade?}

I think the principles of palliative care have not changed. I think they are still looking at the holistic care of patients with terminal and lifelimiting illness, looking holistically at their care, carers and loved ones involved.

I think what has changed is this: first of all cancer palliative care has become an integrative part of medicine. At every oncology center, every oncology department, every oncology multidisciplinary team is expected now to have a palliative care input. Patients' access in hospitals and in the community to specialist oncology has changed.

Another thing that has changed in the natural history of cancer, when I first started in palliative care, when I was first working in the hospices, if you had metastatic breast cancer your prognosis was a matter of months. Now, developments in oncology are such that people will have prognosis of years - it almost takes on a natural history of a chronic disease. As a result, we as palliative care doctors will be involved with patients not just at the end of life but also throughout their cancer journey, dipping in and dipping out more as and when we are needed.

I think the other thing is as people are living longer, there are more comorbidities, more chronic illnesses that we need to be mindful of. As people live longer, they are developing complications that we never realized existed before because people just did not live long enough to get them. So we are seeing a lot more complex things occurring.

I think finally we are trying to be a lot more proactive. There is a much larger emphasis on patients being involved in their preferred places of care, being involved in resuscitation decisions, being empowered to actually make decisions for what they would hope for rather, than the doctor being God who says, "you have this!" We are involving our patients more. I believe those models of care then rub off on our general colleagues and our oncology colleagues. I think the one we have to be aware of as palliative care doctors is that we must also allow the philosophy and the medical knowledge of general physicians rub off on us as well, so learn from our colleagues as much as they learn from us.

Q CAT is a condition that is not normally discussed, despite it being one of the leading causes of death in cancer patients. Can you tell our readers more about the condition \& the risk factors involved?

I think the bottom line is that clotting is a normal part of our bodies healing, but sometimes that process can go wrong. We know that the malignant state, in other words, the presence of cancer, will increase the release of molecules in the blood that increase its coagulability. However, we know that not all cancers are the same; we know that, say, primary brain, primary lung or pancreatic cancer can increase your risk of thrombosis up to 19-fold, while in breast and prostate cancer the risk may be only fourfold. If you then add in aspects such as metastatic spread, the risk of thrombosis can likewise increase up to 19-fold. You then find that treatments we give like chemotherapy and surgery will increase the risk. So you have got all these different factors coming together to increase the risks of blood clots. What I find most interesting here is that some patients will actually present with a deep vein thrombosis or a pulmonary embolus, before 
their diagnosis of the cancer and it is the fact that they have hypercoagulable blood due to the cancer that first leads people to the diagnosis of the cancer.

Another thing that is very important to note is that $52 \%$ of people that get CAT will do so in the first 3 months of diagnosis of cancer. So you have people who are still reeling from their diagnosis of a life-threatening condition and then usually within 3 months of that, usually as they have just started their treatment for it, they will then have another life-threatening condition that is either a deep vein thrombosis or a pulmonary embolus; so the psychological impact of that cannot be underestimated.

\section{Q Can you tell us about the Patient} Experience of Living with Cancer Associated Thrombosis study, which assessed patient experience of living with CAT?

The PELICAN study stands for Patient Experience of Living with Cancer Associated Thrombosis and it is an ongoing study, which is doing the same protocol in a lot of different countries. The one that we have currently published on was from the UK in which we interviewed patients with CAT and analyzed these interviews to see if there were similar experiences.

The main things we found were, first and foremost, that patients found CAT a very distressing experience, which I think people underestimate how upsetting it can be to have. What is more is when they have the diagnosis, the process of managing it tends to be very rushed and they do not have access to information and support.

If you are not getting the information and the explanations you need, you are more likely to consult that 'anarchic library' known as the internet, and there you can find all sorts of frightening information, some of it accurate, some of it inaccurate, so there is an information gap there.

I think the other thing that people find is living with uncertainty is something that really upsets them - they need answers and they need support. It is like being broken bad news a second time. We know that when most people are told they have cancer, you break bad news in a sensitive way; you are making sure that you are not going to be disturbed when it happens, you make sure there is availability of follow-up. We are not doing that when they have a potentially life-threatening clot. I think people are left hanging in the wind.
I think another thing it showed is that patients are incredibly resilient and robust. In terms of the perception that they will not manage having low-molecular-weight heparin injections - for example, people tend to develop their own routines and practices, and normalize the process of self-injecting. A lot of people will make habits out of it and will actually cope very well with it.

What we are looking at now is whether these experiences are common across the world because these are only the experiences of Caucasian Brits that are treated through the National Health Service. How does it differ with Mediterranean people in a semi-private healthcare setting? Or how does it differ in Manila where you may not have access to the same medicines or live in a rural area? Are there common aspects here that we need to be looking at or are there cultural differences?

Q A clear aim of the study is to address subsequent behavioral factors \& psychological distresses following diagnosis of the condition. How important is this aspect of supporting the patient following initial diagnosis \& treatment of the condition? I think it is absolutely essential and I think what we need to be doing is preempting it. One thing that patients would often tell us in the PELICAN study is that no one warned them of the risk of clots. Most people have only heard that clots occur in people on long haul flights. They are not told that up to $20 \%$ of cancer patients may get clots during their cancer journey. They are made aware of the fact that thrombosis is the major killer of people during chemo. Now these same people when they receive chemotherapy are given every bit of information they need about the risk of febrile neutropenia. They know that if they get certain red-flag symptoms, they are to call a chemotherapy pager number and there will be a pathway for managing sepsis. But these patients are not warned if you become breathless, if your legs become swollen, they should ring ' $x$ ' number. As a result, patients often just assume they are feeling breathless and they assume their legs are swollen because they have cancer and they are having chemotherapy so of course they are feeling unwell. I think preempting and prewarning are important.

I think the other thing is that when these people realize they have a life-threatening condition, that increases the level of anxiety and I think that is a very strong prompt for us for want of better word 'normalizing' CAT. You do not see 
people who have had a sepsis experiencing the same levels of ongoing anxiety and distress about it as you do with thrombosis. This is because they are told when you have chemotherapy, it is not unusual to get septic and this is what can be about it. I think we should advise our patients about thrombosis and say this is a normal thing that occurs and is important to treat because it is potentially very dangerous. Nevertheless, if it is seen as part and parcel of the cancer journey rather than a life-threatening complication, I think of the level of distress would be lower. Now at the moment when we give patient information we need to make sure they have got questions they can ask that can be answered and at the moment the process appears to be too rushed.

Interestingly, we have done some other research in noncancer patients, which shows that patients who have experienced a clot will develop ongoing anxiety and distress, and proportion of them will develop post-traumatic stress disorder. One of the reasons that triggers post-traumatic stress disorder is an unexpected threat to life and then living with the unknowing of whether that threat is likely to return so it is that uncertainty which causes a lot of the distress.

Q The study highlights several limitations in the patient understanding of this condition. How can we overcome these limitations \& improve supportive care?

I think there are a few angles here. I think the first one is you have got to get your medical and nursing colleagues on side. We have often called CAT a bit of a 'Cinderella' symptom as people do not always think of it when people are experiencing the symptoms. so people do not see the importance of it. Essentially, there needs to be a paradigm shift, an attitude change, in clinicians first of all.

Second, we need to give patients information but in a way they can accept it. If we talk about the majority of people who develop a clot, they will get it early on in the cancer diagnosis and early on in chemotherapy. We have to be very mindful that these people are getting bombarded with information at a time when they are not going to retain it all. One thing I am particularly proud of is some work that Anticoagulation Europe has produced, which is a video called, 'Blood Clots, Cancer and You'. This is a very gentle but informative video, which explains to patients, the signs and symptoms of clots very clearly within the patients' cancer experience. It is the sort of thing where you do not have to force the information down to the patient's throat. We have been purchasing tablets with the video, so that when patients are receiving chemotherapy they can chose to watch it or not watch it as they feel so inclined. The information, therefore, is there for them if they want it, but it is not just in a pile of leaflets that they may or may not read.

There need to be novel ways of getting this information across to patients. Just because you have told someone this information once does not mean they have absorbed it or understood it. Sometimes you need to drip feed people information over a period of time.

\section{Q What resources are available to clinicians to drive awareness of CAT?}

I think you need to chip away, as the developments we have had have not been done overnight. It has been though persistence and working alongside our oncology colleagues. I think what we have to remember, it is easy for me as someone who has an interest in blood clots and cancer to think that it is the most important thing in the world and of course I would. But my oncology colleagues have to deal with a lot of complications of cancer; they have to deal with febrile neutropenia, they have got to deal with nausea, plantar-palmar syndrome, mucositis, problems with nutrition, psychological issues as well as other issues regarding the complications of the cancer spread.

This is not a willful ignoring by our colleagues. They simply have a lot of different issues they are dealing with all the time. There has to be a persistent aim to increase awareness and potentially having a champion in that area that has a interest in CAT. This is a rapidly changing and developing field and I think it is quite sensible to have a clinician with a specialist interest in it, who you can refer the more complex patients to. Is that going to be a hematologist? Is it going to be a supportive care clinician who has that interest? I do not think it matters, so long as there is someone who can be the go to guy for CAT.

\section{Q Can you tell our readers about your current research \& other ongoing studies?}

First of all I am continuing the PELICAN study. We have analyzed data from Spain, which we are writing up at the moment and we are also analyzing data from Canada. We have still got data from France coming in and we have opened recently in New Zealand. We have also got other 
countries that are opening such as Manila, Singapore and also The Netherlands. We have now got quite a few countries still going so that will be very interesting.

We are also working with the Haemostasis and Biomarker Research Unit in Swansea University (UK) where they have developed a technique of measuring a fractal analysis of the incipient blood clot, basically the baby blood clot as it forms. As a result the team are able to look at the microstructure of the clot and identify that it changes depending on the stage of a cancer.

We know that patients with early-stage cancer have a less dense clot than those with a more malignant cancer and we have got data that shows that lung cancer patients with stage IV cancer form a clot about four-times as dense as the clots formed by a healthy person. So it shows that cancer patients not only make clots more regularly but they also make clots that are much more resistant to lysis and treatment. So what we are planning to do now is see what happens to clot microstructure when you give these patients chemotherapy, because it would be very useful to see how much it changes depending on the type of chemotherapy you give. Then, arguably, you could identify which patients are at greater risk of developing thrombosis during chemotherapy, and target them appropriately with thromboprophylaxis.

There are other studies we are doing such as the 'Blood Clots, Cancer and You' video that we are introducing in one of our hospitals. While it is great to introduce these new gadgets, I think it is important to evaluate whether or not they actually affect outcomes. We now have a study that is going to look at the impact of this scheme on patient behaviors, staff behaviors and time taken to present with symptoms of thrombosis.

\section{Q Where do you hope to see the} management of CAT \& cancer supportive care in 10 years' time?

What I would hope is that we will have got to a point where, rather than looking at CAT as a single entity, we have recognized that it is a highly heterogeneous state. The thrombosis that is formed by someone receiving adjuvant chemotherapy for breast cancer is going to be a different sort of clot to a patient with stage IV pancreatic cancer receiving palliative chemotherapy. They are very different of the same problem but may accordingly require different approaches to their management.
Most of the studies that have been done thus far in the treatment and the prevention of CAT have looked at cancer as a single homogeneous entity, but when you put in pancreatic cancer patients alongside breast and prostate patients they are highly heterogeneous, so I think there is going to be a far more individualized approach.

I would also like to think that there is more patient involvement in the decision. What we have now got is a breadth of anticoagulants with different properties and I think there should be an opportunity to tailor-make the prophylaxis and the treatment plans for these patients. It is OK for us now that we can look at the molecular make-up for certain tumors and identify the most appropriate chemotherapy according to genetic markers, but we are still treating all different cancer thromboses the same at the moment.

\section{Q Closing statement}

I would say that CAT is a phenomenon that is not going to go away; people are living longer and we know that age is a risk factor. Likewise people are living longer with metastatic disease and are receiving more and more chemotherapy, palliative and adjuvant. All the factors for thrombosis are increasing so it is likely that as clinics we are going to see more and more CAT, so it is not an issue that we can pretend is going to go away. It is likely to become a very common part of our clinical practice. Since it is a major killer and a major cause of psychological distress and physical morbidity, I think it is important that we all get together and recognize that this is something we have to take seriously and be proactive about.

\section{Disclaimer}

The opinions expressed in this interview are those of the interviewee and do not necessarily reflect the views of Future Medicine Ltd.

Financial \& competing interests disclosure

In the past 2 years, S Noble has undertaken speaker engagements and advisory board roles for Leo Pharma, Pfizer, Boehringer Ingelheim and Bristol-Myers Squibb. He has taken no honoraria for these. S Noble has no other relevant affiliations or financial involvement with any organization or entity with a financial interest in or financial conflict with the subject matter or materials discussed in the manuscript apart from those disclosed.

No writing assistance was utilized in the production of this manuscript. 